\title{
Nonlinear Effects in Three-minute Oscillations of the Solar Chromosphere. I. An Analytical Nonlinear Solution and Detection of the Second Harmonic
}

\author{
Jongchul Chae ${ }^{1}$ and Yuri E. Litvinenko ${ }^{2}$ \\ ${ }^{1}$ Astronomy Program, Department of Physics and Astronomy, Seoul National University, Seoul 08826, Korea \\ ${ }^{2}$ Department of Mathematics, University of Waikato, P. B. 3105, Hamilton 3240, New Zealand \\ Received 2017 May 5; revised 2017 June 12; accepted 2017 June 21; published 2017 July 31
}

\begin{abstract}
The vertical propagation of nonlinear acoustic waves in an isothermal atmosphere is considered. A new analytical solution that describes a finite-amplitude wave of an arbitrary wavelength is obtained. Although the short- and long-wavelength limits were previously considered separately, the new solution describes both limiting cases within a common framework and provides a straightforward way of interpolating between the two limits. Physical features of the nonlinear waves in the chromosphere are described, including the dispersive nature of lowfrequency waves, the steepening of the wave profile, and the influence of the gravitational field on wavefront breaking and shock formation. The analytical results suggest that observations of three-minute oscillations in the solar chromosphere may reveal the basic nonlinear effect of oscillations with combination frequencies, superposed on the normal oscillations of the system. Explicit expressions for a second-harmonic signal and the ratio of its amplitude to the fundamental harmonic amplitude are derived. Observational evidence of the second harmonic, obtained with the Fast Imaging Solar Spectrograph, installed at the $1.6 \mathrm{~m}$ New Solar Telescope of the Big Bear Observatory, is presented. The presented data are based on the time variations of velocity determined from the Na I $\mathrm{D}_{2}$ and $\mathrm{H} \alpha$ lines.
\end{abstract}

Key words: hydrodynamics - Sun: atmosphere - Sun: chromosphere - Sun: oscillations - waves

\section{Introduction}

The solar chromospheric plasma is often observed to execute an up-and-down motion with a period of about three minutes. It is generally accepted that these three-minute (3 minute) oscillations indicate the presence of vertically propagating acoustic waves with frequencies slightly above the acoustic cutoff $\omega_{0}$ in a gravitationally stratified medium (e.g., Felipe et al. 2010; Chae \& Goode 2015; Wiśniewska et al. 2016). The key physical idea, originally formulated by Fleck \& Schmitz (1991) and Kalkofen et al. (1994), is that the waves constitute a response of the solar atmosphere to a general velocity disturbance at its base. The atmospheric response to various types of continuous and pulsed driving at the lower photospheric boundary had been described analytically (e.g., Sutmann et al. 1998; Petukhov \& Petukhov 2001) and numerically (e.g., Kalkofen et al. 2010; Fawzy \& Musielak 2012). Because a single pulse or a periodic perturbation at the boundary generally lead to a decaying chromospheric signal at the cut-off frequency, the observed persistent 3 minute oscillations are typically assumed to be driven by a sequence of random pulses (Sutmann et al. 1998).

As emphasized by Chae \& Goode (2015), the predominance of 3 minute oscillations in the chromosphere can be explained by the dispersive nature of acoustic waves in the stratified medium. At heights far above the base, the waves at frequencies just above the acoustic cutoff dominate. Indeed the waves with frequencies $\omega<\omega_{0}$ become evanescent, and the high-frequency waves with $\omega \gg \omega_{0}$ propagate at the sound speed and hence quickly move away. By contrast, the wave packets with frequencies $\omega \gtrsim \omega_{0}$ propagate with group speeds lower than the sound speed. Consequently, the propagating waves do not differ greatly from standing waves and appear to remain in the atmosphere. In this regard, both the storage and transmission of wave energy of the 3 minute oscillations takes place in the chromosphere. The power for the upwardpropagating waves can be supplied, for instance, by a series of local impulsive disturbances of vertical extent larger than twice the pressure scale height (Chae \& Goode 2015).

How do the acoustic waves with frequencies $\omega \gtrsim \omega_{0}$ evolve as they propagate upward through the chromosphere? Recent investigations strongly suggest that the waves propagating in the upper chromosphere above sunspots are highly nonlinear (e.g., Chae et al. 2014; Tian et al. 2014). The time variation of the observed velocity at a fixed point often displays a sawtooth pattern, characterized by a sudden appearance of a rapid upward motion, followed by gradual deceleration and transition to downward motion. This pattern implies the presence of an upwardly propagating shock. Physically, the wave velocity amplitude increases with height as the background density decreases. When the amplitude reaches a significant fraction of the sound speed, the velocity profile steepening leads to wave breaking and shock formation. The effect was described analytically (Ostrovskii 1963; Naugolnykh \& Ostrovsky 1998), though the description was valid only in a high-frequency limit $\omega \gg \omega_{0}$ and could not describe the physically relevant case $\omega \simeq \omega_{0}$ of dispersive low-frequency waves. Analytical description of a nonlinear acoustic wave before wave breaking was also achieved for nondispersive high-frequency waves (Nayfeh 1975).

Litvinenko \& Chae (2017) noted new opportunities for observing the 3 minute oscillations and hence the need for a theoretical description of finite-amplitude dispersive acoustic waves with frequencies $\omega \gtrsim \omega_{0}$, which extends the traditional analysis of linearized equations. Litvinenko \& Chae (2017) presented analytical solutions for finite-amplitude wave propagation in both dispersive (long-wavelength) and nondispersive (short-wavelength) limits. The solutions provided a simple description of the basic nonlinear effect of a secondharmonic generation at twice the driving wave frequency. 
The effect appears to have been noted in a numerical study of chromospheric oscillations (Kalkofen et al. 1994), and estimates based on the new solutions suggest that a signal at frequency $2 \omega$ should be detectable by a number of currently operating solar instruments (Litvinenko \& Chae 2017).

Motivated by these considerations, the purpose of this paper is twofold. First, we present a new solution for a nonlinear acoustic wave in an isothermal atmosphere. The solution not only yields the short- and long-wavelength limits that we previously considered (Litvinenko \& Chae 2017) but also allows us to interpolate between the two limits and describe a finite-amplitude wave for the case of an arbitrary frequency of the perturbation at the photospheric boundary. We use the solution to infer the formation of shock waves and to investigate the generation of a second harmonic from the 3 minute oscillations. Second, we present observational evidence of the second harmonic, obtained with the Fast Imaging Solar Spectrograph (FISS), installed at the $1.6 \mathrm{~m} \mathrm{New}$ Solar Telescope (NST) of the Big Bear Observatory (Kwak et al. 2016). We apply the new solution to quantitatively analyze the observed time variations of velocity determined from the $\mathrm{Na} \mathrm{I}_{2}$ line and the $\mathrm{H} \alpha$ line.

\section{An Analytical Nonlinear Solution for a Simple Wave}

\subsection{Basic Equations and Approximations}

We consider the adiabatic upward propagation of a simple acoustic wave in an isothermal atmosphere in hydrostatic equilibrium. The property of medium is described by three independent parameters: the sound speed $c_{0}$, the gravitational acceleration $g$, and the ratio of specific heats $\gamma$. The gravitational scale height of the background atmospheric pressure $p_{0}(z)$ (and density $\left.\rho_{0}(z)\right)$ is given by

$$
H=\frac{c_{0}^{2}}{\gamma g},
$$

and the acoustic cutoff frequency is defined as

$$
\omega_{0}=\frac{\gamma g}{2 c_{0}}
$$

where $z$ is the vertical coordinate.

It is assumed that the wave is driven by a harmonic perturbation of angular frequency $\omega$ at the lower boundary $z=0$ :

$$
v(0, t)=v_{0} \sin (\omega t),
$$

where $t$ is time and $v$ is the velocity along the $z$ axis. The frequency $\omega$ must be greater than $\omega_{0}$ in order for the waves to propagate upward.

As previously described by Litvinenko \& Chae (2017), the vertical propagation of plane adiabatic acoustic waves in the presence of gravity is governed by the momentum equation

$$
\rho \frac{D v}{D t}=\rho\left(\frac{\partial v}{\partial t}+v \frac{\partial v}{\partial z}\right)=-\frac{\partial p}{\partial z}-\rho g,
$$

the continuity equation

$$
\frac{D \rho}{D t}=-\rho \frac{\partial v}{\partial z}
$$

and the entropy equation

$$
\frac{D p}{D t}=c^{2} \frac{D \rho}{D t}
$$

where $v=v(z, t)$ is the velocity along the $z$ axis, $\rho$ and $p$ are the fluid density and pressure in the wave, and $c$ is the local sound speed:

$$
c^{2}=\frac{\gamma p}{\rho}
$$

It was shown by Litvinenko \& Chae (2017) that these equations can be combined to yield

$$
\frac{D^{2} v}{D t^{2}}-c^{2} \frac{\partial^{2} v}{\partial z^{2}}+\gamma g \frac{\partial v}{\partial z}=-\gamma \frac{\partial v}{\partial z} \frac{D v}{D t}
$$

Equation (8) has no exact analytical solution, except the Riemann wave in the gravity-free case $g=0$ (e.g., Lighthill 1978). To make further analytical progress, in the following, we consider moderately nonlinear waves, and we use a second-order approximation in which only linear and quadratic terms of $v$ and its derivatives are retained in Equation (8):

$$
\begin{gathered}
\frac{\partial^{2} v}{\partial t^{2}}-c_{0}^{2} \frac{\partial^{2} v}{\partial z^{2}}+\frac{c_{0}^{2}}{H} \frac{\partial v}{\partial z}=c_{0}^{2} q \frac{\partial^{2} v}{\partial z^{2}} \\
-2 v \frac{\partial^{2} v}{\partial z \partial t}-(1+\gamma) \frac{\partial v}{\partial z} \frac{\partial v}{\partial t} .
\end{gathered}
$$

Here the quantity

$$
q=\frac{c^{2}}{c_{0}^{2}}-1 \approx \frac{p_{1}}{p_{0}}-\frac{\rho_{1}}{\rho_{0}}
$$

where $p_{1}=p-p_{0}$ and $\rho_{1}=\rho-\rho_{0}$, is governed by the linearized equation

$$
\frac{\partial q}{\partial t}=-(\gamma-1) \frac{\partial v}{\partial z}
$$

(see the derivation of Equations (31)-(33) in Litvinenko \& Chae 2017 for details).

We find it convenient to introduce the dimensionless variables defined by

$$
\tilde{z} \equiv \frac{z}{2 H} ; \quad \tilde{v} \equiv \frac{v}{c_{0}} ; \tilde{t} \equiv t \omega_{0} ; \tilde{\omega} \equiv \frac{\omega}{\omega_{0}}
$$

but it is cumbersome to keep the tilde superscript in all of the equations. Thus we maintain the same notations $z, v, t, \omega$, but from now on identify them with the dimensionless parameters $\tilde{z}, \tilde{v}, \tilde{t}, \tilde{\omega}$ defined above, unless specified otherwise. Thus we can rewrite Equations (9) and (11) in the dimensionless form:

$$
\frac{\partial^{2} v}{\partial t^{2}}-\frac{\partial^{2} v}{\partial z^{2}}+2 \frac{\partial v}{\partial z}=q \frac{\partial^{2} v}{\partial z^{2}}-2 v \frac{\partial^{2} v}{\partial z \partial t}-(1+\gamma) \frac{\partial v}{\partial z} \frac{\partial v}{\partial t},
$$

$$
\frac{\partial q}{\partial t}=-(\gamma-1) \frac{\partial v}{\partial z}
$$




\subsection{Linear Acoustic Waves}

In the small-amplitude limit, Equation (13) simplifies to the linear equation

$$
\frac{\partial^{2} v}{\partial t^{2}}-\frac{\partial^{2} v}{\partial z^{2}}+2 \frac{\partial v}{\partial z}=0
$$

By introducing $u$, defined by

$$
v=e^{z} u,
$$

we can rewrite Equation (15) as

$$
\frac{\partial^{2} u}{\partial t^{2}}-\frac{\partial^{2} u}{\partial z^{2}}+u=0
$$

which is the familiar form of the equation describing linear acoustic waves in an atmosphere.

We seek the solution of the form

$$
u(z, t)=v_{0} \sin (\omega t-k z)
$$

or

$$
v(z, t)=v_{0} e^{z} \sin (\omega t-k z),
$$

where $k$ is a dimensionless wavenumber. We take $k$ to be positive for the consideration of upwardly propagating waves. Equation (19) obviously satisfies the boundary condition in Equation (3). Substitution of Equation (18) into (17) leads to the dispersion relation:

$$
k=\sqrt{\omega^{2}-1} .
$$

The dimensionless phase speed is given by

$$
c_{p}=\frac{\omega}{k}=\frac{\omega}{\sqrt{\omega^{2}-1}},
$$

where the sign of $k$ is taken to be positive to represent upwardly propagating waves.

We define the waves with $k>1$ as short waves, and those with $k<1$ as long waves. Equivalently, the high-frequency waves $(\omega>\sqrt{2})$ are short waves, and the low-frequency waves $(1<\omega<\sqrt{2})$ are long waves. Physical properties of the short and long waves qualitatively differ from each other. Short waves are almost nondispersive. Their phase speed $c_{p}$ is close to 1 and depends on $\omega$ only weakly. On the contrary, very long waves are highly dispersive. Their phase speed significantly exceeds 1 and strongly depends on $\omega$. The commonly observed 3 minute oscillations correspond to long waves. Because very long waves have very small values of $k$, they appear to an observer as standing waves.

\subsection{A Nonlinear Wave Solution}

We seek a nonlinear solution in the form

$$
v(z, t)=v_{0} e^{z} \sin (\omega t-k z+b(z) \omega v),
$$

which is a modification of Equation (19), mimicking the textbook form of an implicit solution for a nonlinear wave in the absence of gravity (e.g., Lighthill 1978). Then the problem reduces to finding a new dimensionless function $b(z)$ that makes Equation (22) an exact or approximate solution of Equations (13) and (14). We require that the nonlinear solution in Equation (22) reduces to the linear solution in Equation (19) for small $z$. Consequently, we impose the boundary condition

$$
b(0)=0 .
$$

By introducing the phase function $\theta$ defined by

$$
\theta(z, t) \equiv \omega t-k z+b(z) \omega v,
$$

the velocity amplitude

$$
v_{1}(z) \equiv v_{0} e^{z},
$$

and the function $f$

$$
f(\theta)=\sin \theta,
$$

we can rewrite Equation (22) as

$$
v=v_{1}(z) f(\theta) .
$$

By differentiating this equation with respect to $t$ and solving for $\partial v / \partial t$, we obtain

$$
\frac{\partial v}{\partial t}=f^{\prime} X\left(1-f^{\prime} X b\right)^{-1},
$$

with the dimensionless velocity ampltitude parameter $X$ defined by

$$
X \equiv \omega v_{1},
$$

where $f^{\prime}$ refers to the derivative of $f$ with respect to $\theta$. We assume $\left|f^{\prime} X b\right|<1$ and use the expansions

$$
\left(1-f^{\prime} X b\right)^{-1}=1+f^{\prime} X b+f^{\prime 2} X^{2} b^{2}+\cdots
$$

and

$$
\frac{\partial v}{\partial t}=f^{\prime} X+f^{\prime 2} X^{2} b+\cdots
$$

up to second-order terms in $X$. Ignoring the higher-order terms is consistent with the accuracy of our approximate Equation (13). Similarly, on differentiating Equation (22) with respect to $z$, we obtain the expansion

$$
\frac{\partial v}{\partial z}=\frac{1}{\omega}\left[X\left(f-f^{\prime} k\right)+X^{2}\left(f f^{\prime}\left(b+b^{\prime}\right)-f^{\prime 2} k b\right)+\cdots\right]
$$

up to second-order terms in $v_{0}$, where $b^{\prime}$ refers to the derivative of $b$ with respect to $z$. The above expressions yield

$$
\frac{\partial \theta}{\partial t}=\omega+\omega b \frac{\partial v}{\partial t}=\omega\left(1+f^{\prime} X b+\cdots\right)
$$

and

$$
\begin{aligned}
\frac{\partial \theta}{\partial z} & =-k+\omega b^{\prime} v+\omega b \frac{\partial v}{\partial z} \\
& =-k+X\left(f\left(b+b^{\prime}\right)-f^{\prime} k\right)+\cdots
\end{aligned}
$$

Now we use Equations (32) and (33) to integrate Equation (14) with the same accuracy:

$$
q=\int \frac{\partial q}{\partial t}\left(\frac{\partial \theta}{\partial t}\right)^{-1} d \theta=(\gamma-1) \frac{1}{\omega^{2}} X\left(f^{\prime}+f k\right)+\cdots
$$

Next we substitute our results into Equation (13). The firstorder terms of $v_{1}$ yield

$$
\left(-\omega^{2}+1+k^{2}\right) \frac{X}{\omega} f=0,
$$


which leads to the linear wave solution specified by Equation (20). On collecting all the terms in Equation (13) that are quadratic in $v_{1}$, we obtain after some algebra:

$$
\begin{aligned}
& \frac{X^{2}}{\omega}\left[\left(f f^{\prime}\right)^{\prime}\left(2 b+2 b^{\prime}\right) k-f f^{\prime}\left(4 b+2 b^{\prime}+b^{\prime \prime}\right)\right] \\
& =\frac{X^{2}}{\omega}\left[\left(f f^{\prime}\right)^{\prime}\left((\gamma+1)-2(\gamma-1) \frac{1}{\omega^{2}}\right)\right. \\
& \left.\quad \times k-f f^{\prime}\left(4 \gamma-4(\gamma-1) \frac{1}{\omega^{2}}\right)\right] .
\end{aligned}
$$

This simplifies to the following equation for $b\left(z_{1}\right)$ :

$$
\left(f f^{\prime}\right)^{\prime}\left(b+b^{\prime}-\alpha\right) k-2 f f^{\prime}\left(b+\frac{1}{2} b^{\prime}+\frac{1}{4} b^{\prime \prime}-\beta\right)=0,
$$

where

$$
\alpha=\frac{1}{2}(\gamma+1)-(\gamma-1) \frac{1}{\omega^{2}}
$$

and

$$
\beta=\gamma-(\gamma-1) \frac{1}{\omega^{2}} .
$$

Because $f f^{\prime}$ and $\left(f f^{\prime}\right)^{\prime}$ are linearly independent, the coefficients of both $f f^{\prime}$ and $\left(f f^{\prime}\right)^{\prime}$ must vanish in order for Equation (38) to be valid. Consequently, the two independent differential equations

$$
b+\frac{d b}{d z}-\alpha=0
$$

and

$$
b+\frac{1}{2} \frac{d b}{d z}+\frac{1}{4} \frac{d^{2} b}{d z^{2}}-\beta=0,
$$

must hold for $b$ concurrently. We find it impossible to determine an exact solution $b(z)$ that satisfies the above equations, which may be partly attributed to the approximations adopted to derive the wave equations. Alternatively we seek an approximate solution of the form

$$
b(z)=W b_{S}(z)+(1-W) b_{L}(z),
$$

where $b_{S}(z)$ is the short-wave (large $k$ ) solution satisfying Equation (41) and $b_{L}(z)$ is the long-wave (small $k$ ) solution satisfying Equation (42) and $W$ is an interpolation parameter chosen so that Equation (38) is satisfied with a maximum accuracy.

Making use of the boundary condition in Equation (23), we solve Equation (41) to obtain the short-wave solution:

$$
b_{S}(z)=\alpha\left(1-e^{-z}\right) \text {, }
$$

and we solve Equation (42) to obtain the long-wave solution:

$$
b_{L}(z)=\beta\left[1-\left(\cos (\sqrt{3} z)+\frac{1}{\sqrt{3}} \sin (\sqrt{3} z)\right) e^{-z}\right] .
$$

Note for clarity that a second integration constant in $b_{L}$ is specified by the condition that the function $b_{L}$ be as close as possible to a monotonically increasing function. The condition follows from the requirement that the velocity profile $v$ be

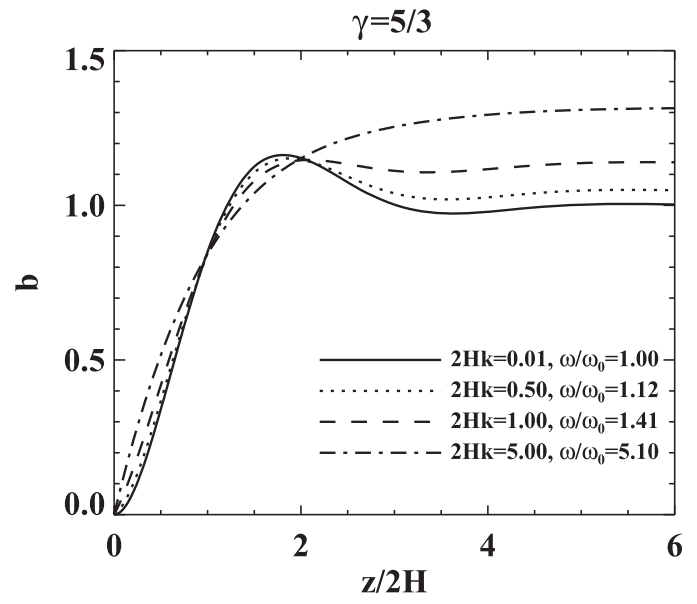

Figure 1. Plots of $b(z)$ for different values of $k$.

approximated by a single-valued function. (The function $b_{S}$ is also monotonic.) Specifically, we choose an integration constant to minimize the difference between the local maximum and minimum values of the function $b_{L}$ and therefore to maximize the height $z_{m}$, where the first local maximum occurs. It is easy to verify that the procedure leads to $b_{L}^{\prime}(0)=0$ and $z_{m}=\pi / \sqrt{3}$.

Finally, by minimizing the functional

$$
\begin{aligned}
F(W) \equiv & \int_{0}^{\infty}\left[\left(b+b^{\prime}-\alpha\right)^{2} k^{2}\right. \\
& \left.+\left(b+\frac{1}{2} b^{\prime}+\frac{1}{4} b^{\prime \prime}-\beta\right)^{2}\right] d z
\end{aligned}
$$

$$
\begin{aligned}
= & (1-W)^{2} \int_{0}^{\infty}\left(b_{L}+b_{L}^{\prime}-\alpha\right)^{2} k^{2} d z+W^{2} \int_{0}^{\infty} \\
& \times\left(b_{S}+\frac{1}{2} b_{S}^{\prime}+\frac{1}{4} b_{S}^{\prime \prime}-\beta\right)^{2} d z,
\end{aligned}
$$

we obtain the expression of $W$

$$
W=\frac{\int_{0}^{\infty}\left(b_{L}+b_{L}^{\prime}-\alpha\right)^{2} k^{2} d z}{\int_{0}^{\infty}\left(b_{L}+b_{L}^{\prime}-\alpha\right)^{2} k^{2} d z+\int_{0}^{\infty}\left(b_{S}+\frac{1}{2} b_{S}^{\prime}+\frac{1}{4} b_{S}^{\prime \prime}-\beta\right)^{2} d z}
$$

These expressions of the definite integrals are unwieldy but they are straightforward to evaluate numerically. In fact, we found that $W$ is fairly well approximated by a very simple rational function

$$
W \approx \frac{\omega^{2}-1}{\omega^{2}-0.283}
$$

which becomes zero when $\omega=1$ and unity when $\omega \gg 1$.

Summing up, the set of Equations (39), (40), (43), (44), (45), and (49) fully specifies the solution for $b(z)$ when $\omega$ is specified. Figure 1 shows the solution for $b(z)$ obtained for several representative cases of short and long waves. 

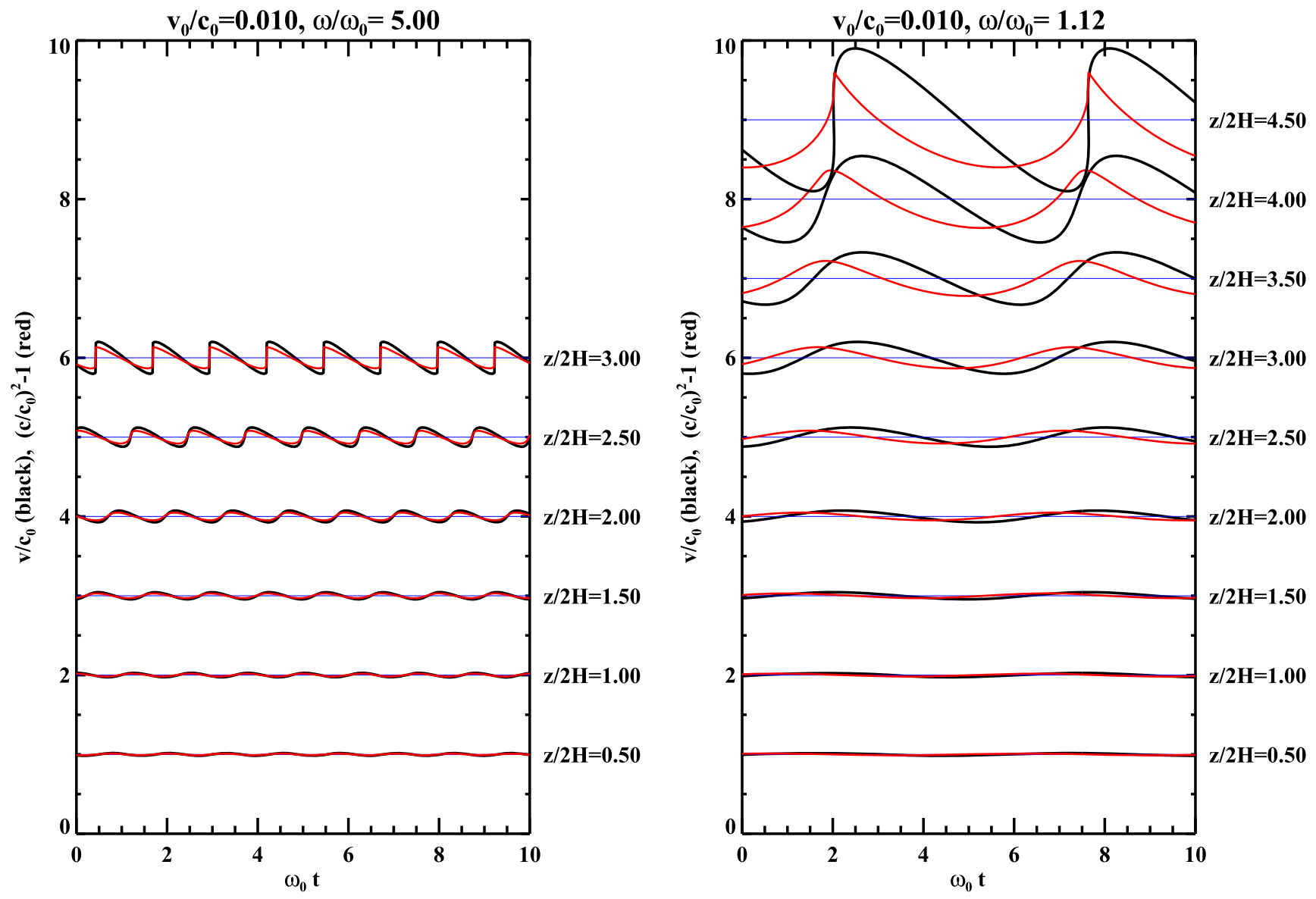

Figure 2. Velocity and temperature oscillations at different heights for short-period driving (left) and long-period driving (right).

\section{Features of the Solution}

\subsection{Short-wavelength and Long-wavelength Regimes}

In the short-wave limit, we have $k \gg 1, W \approx 1$, and $b \approx b_{S}$. It follows that $k \approx \omega$ and $\alpha \approx(\gamma+1) / 2$. Thus we can rewrite the formal solution in Equation (22) as follows.

$$
v=v_{0} e^{z} \sin (\omega t-\omega z+b(z) \omega v)
$$

where

$$
b(z) \approx b_{S}(z) \approx \frac{(\gamma+1)}{2}\left(1-e^{-z}\right) .
$$

The solution in this limit coincides with the short-wave solution presented by Litvinenko \& Chae (2017).

In the opposite long-wavelength limit, we have $k \ll 1$, $W \approx 0$, and $b \approx b_{L}$. It follows that $\beta \approx 1$. Consequently,

$$
v=v_{0} e^{z} \sin \left(\omega t-\sqrt{\omega^{2}-1} z+b \omega v\right),
$$

where

$$
b(z) \approx b_{L}(z) \approx 1-\left(\cos \sqrt{3} z+\frac{1}{\sqrt{3}} \sin \sqrt{3} z\right) e^{-z} .
$$

Figure 2 shows the solutions $v$ and $q$ in two cases: shortperiod driving and long-period driving. The zero-velocity phases move at the sound speed in high-frequency waves, but they move at much faster speeds in low-frequency waves. Figure 2 also illustrates that the velocity amplitude increases and the velocity profile steepens with height, implying that the high-frequency (short) waves become shock waves at lower heights than the low-frequency (long) waves. Note that local temperature, as measured by $q$, fluctuates with time because of adiabatic compression and rarefaction. Temperature fluctuation is in phase with velocity fluctuation in the short waves, but is not in phase in the long waves. When shocks form, temperature reaches a peak just after the shock front in both the waves.

\subsection{Wavefront Breaking and Shock Formation}

As a nonlinear acoustic wave propagates upward, the wave profile steepens and eventually wave breaking takes place. This occurs at a height $z_{\mathrm{wb}}$, where the maximum value of $\partial v / \partial t$ in Equation (28) becomes infinite, which yields

$$
1-b\left(z_{\mathrm{wb}}\right) X\left(z_{\mathrm{wb}}\right)=0 .
$$

By solving Equations (25), (43), (41), (42), and (54), one can determine $z_{\mathrm{wb}}$ as a function of $\omega$ and $v_{0}$. We found that the height $z_{\mathrm{wb}}$ is practically determined by the dimensionless driving parameter $X_{0}$, defined by

$$
X_{0} \equiv X(0)=\omega v_{0} \text {. }
$$

The value of $z_{\mathrm{wb}}$ increases with $1 / X_{0}$. This characteristic is well identified from Figure 3. Even though the same value of $X_{0}$ results in different values of $z_{\mathrm{wv}}$ depending on $\omega$, the difference is not significant. We find that the short-wave solution

$$
z_{\mathrm{wb}, \mathrm{s}}=\ln \left(1+\frac{2}{\gamma+1} \frac{1}{X_{0}}\right)
$$



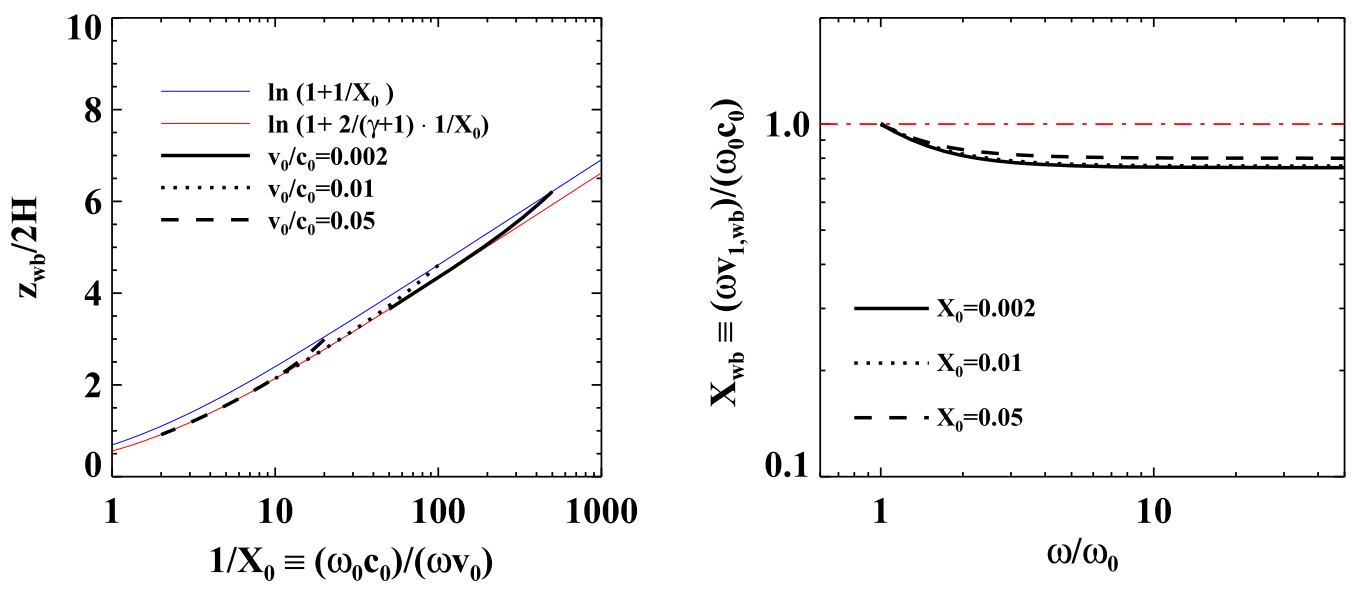

Figure 3. Dependence of the dimensionless height of shock formation $z_{\mathrm{wb}}$ (left) and velocity amplitude parameter $X_{\mathrm{wb}}\left(\right.$ right) on $1 / X_{0}$.

is applicable to $\omega \gg 1$ and the long-wave solution

$$
z_{\mathrm{wb}, \mathrm{L}} \approx \ln \left(1+\frac{1}{X_{0}}\right)
$$

to $\omega \approx 1$. What is important is that the difference between these two is not large. For example, for the same value of $X_{0}=0.01$ the short-wave solution gives the estimate $z_{\mathrm{wb}}=4.33$, while the long-wave solution gives a slightly larger value 4.62 , so that the relative difference is less than $10 \%$.

From Equation (54), we can determine $X$ at the height of shock formation:

$$
X_{\mathrm{wb}} \equiv X\left(z_{\mathrm{wb}}\right)=\frac{1}{b\left(z_{\mathrm{wb}}\right)}
$$

once $z_{\mathrm{wb}}$ is known. The right panel of Figure 3 shows that $X_{\mathrm{wb}}$ is close to 1 , weakly depending on both $\omega$ and $X_{0}$. In the the short-wave limit, by combining Equations (52) and (56), we obtain

$$
X_{\mathrm{wb}}=X_{0}+\frac{2}{\gamma+1} \approx \frac{2}{\gamma+1}
$$

where the last approximation holds when $X_{0} \ll 1$. Meanwhile, in the long-wave limit, from Equations (53) and (57), we obtain

$$
X_{\mathrm{wb}}=1 \text {, }
$$

which, interestingly, is independent of $X_{0}$.

\subsection{Generation of a Second Harmonic}

As an acoustic wave propagates upward and its velocity amplitude increases to compensate for the decrease of the background density, nonlinear effects should come into play. A nonlinear oscillating system generally exhibits oscillations with combination frequencies, superposed on the normal oscillations of the system (Landau \& Lifshitz 1969). As an acoustic wave, generated by a driver at a frequency $\omega$, evolves nonlinearly, the second harmonic of frequency $2 \omega$ is generated as a result of nonlinear interactions expressed as the quadratic terms of perturbations in the wave equation. Stronger nonlinearities will lead to higher-order harmonics as well. Studies of the nonlinear development of acoustic waves are thus interesting because solar observations may reveal the basic nonlinear effect of generating the high-frequency components from lowfrequency ones.
Analytical and numerical studies suggest that the basic nonlinear effect of the second-harmonic excitation may produce observable features at double frequency $2 \omega$ in the solar atmosphere (Kalkofen et al. 1994; Petukhov \& Petukhov 2001; Litvinenko \& Chae 2017). On expanding the solution in Equation (22) as a series of $v_{1}$, we obtain an explicit expression for the second-harmonic signal:

$$
v(z, t)=v_{1}(z)\left[\sin (\omega t-k z)+R_{2} \sin (2(\omega t-k z))+\cdots\right],
$$

where the amplitude ratio between the second and fundamental harmonics is given by

$$
R_{2}=\frac{1}{2} v_{1}(z) \omega b(z) .
$$

When $k \ll 1$ and $z \simeq 1$, these expressions agree with Equation (39) of our previous, more limited analysis of dispersive long-wavelength waves (Litvinenko \& Chae 2017). When $z \ll 1$, the expressions differ somewhat because of a different method employed to derive an approximate solution. Solutions obtained by both methods satisfy the same boundary condition at $z=0$.

\subsection{Model of Nonlinear Velocity Oscillation}

Making use of Equations (22), (24), and (62), we obtain the mathematical model of $v(t)$ at a fixed position

$$
v(t)=v_{1} \sin \left(\omega t+\frac{2 R_{2}}{v_{1}} v(t)-\phi\right)
$$

with the four free parameters: $v_{1}, \omega, R_{2}$, and $\phi$ to be determined from the model fitting. This equation is an implicit function for $v(t)$. At each $t$, once a value of $v$ is specified as the input, the refined estimate of $v$ is obtained from the right-hand side as the output, which becomes the input for the next calculation. This process is repeated until the input and the output become practically the same. It is convenient to start the iteration with the initial guess of $v$ given by the sum of the fundamental component and the second-harmonic component as in Equation (61).

Equation (63) serves as an analysis tool of nonlinear velocity oscillation data. Each parameter has a clear physical meaning; $v_{1}$ and $\omega$ are the amplitude and angular frequency of 
Table 1

Typical Values of the Parameters for Three-minute Oscillations in a Sunspot Umbra

\begin{tabular}{llll}
\hline \hline & Parameter & Value & Comment \\
\hline Input & $c_{0}$ & $6.5 \mathrm{~km} \mathrm{~s}^{-1}$ & Maltby et al. (1986) \\
& $g$ & $0.274 \mathrm{~km} \mathrm{~s}^{-1}$ & \\
& $\gamma$ & 1.67 & chosen \\
& $z_{0}$ & $38 \mathrm{~km}^{-1}$ & Chae et al. (2017) \\
& $v_{0}$ & $62 \mathrm{~m} \mathrm{~s}^{-1}$ & Chae et al. (2017) \\
& $\omega$ & $0.040 \mathrm{~s}^{-1}$ & Chae et al. (2017) \\
& $\omega_{0}$ & $0.035 \mathrm{rad} \mathrm{s}^{-1}$ & \\
& $H$ & $92 \mathrm{~km}$ & \\
& $\omega / \omega_{0}$ & 1.14 & \\
& $v_{0} / c_{0}$ & 0.01 & \\
& $z_{\mathrm{wb}}+z_{0}$ & $853 \mathrm{~km}^{-1}$ & \\
& $v_{\mathrm{wb}}$ & $5.4 \mathrm{~km} \mathrm{~s}^{-1}$ & \\
& & & \\
\hline
\end{tabular}

fundamental oscillation, $R_{2}$ is the ratio of the second-harmonic amplitude to the fundamental one, and $\phi$ is the phase.

\section{Three-minute Oscillations and the Second Harmonic in Sunspots}

Our theoretical work indicates that the nonlinearity of 3 minute oscillations is measured by the strength of the second harmonic. Litvinenko \& Chae (2017) suggested that this second-harmonic signal should be detectable in the upper chromosphere by an instrument such as the FISS (Chae et al. 2013) installed at the $1.6 \mathrm{~m}$ NST of the Big Bear Observatory. Here we report our detection of the second harmonic from the $\mathrm{H} \alpha$ line and the $\mathrm{Na} \mathrm{I} \mathrm{D}_{2}$ line recorded by the FISS. This report is the first of this kind.

\subsection{Application to Three-minute Oscillations in a Sunspot}

We present the list of the parameters in Table 1 to illustrate how the our solution can be applied to 3 minute oscillations in a sunspot. Maltby et al. (1986) provided three atmospheric models of dark regions in a sunspot umbra. The models indicate that temperature is fairly constant over the heights from 0 to $1000 \mathrm{~km}$ above the surface $\tau_{500 \mathrm{~nm}}=1$. Specifically, the model $\mathrm{M}$ atmosphere in this range can be approximated by an isothermal atmosphere characterized by $c_{0}=6.5 \mathrm{~km}$. It is quite good to assume the constancy of gravitational acceleration in the photosphere and chromosphere, so we have $g=0.274 \mathrm{~km} \mathrm{~s}^{-2}$. Furthermore, we adopt $\gamma=5 / 3$ based on the assumption of adiabatic process of monatomic gas.

Chae et al. (2017) presented observational results of 3 minute oscillations in the photosphere of a sunspot umbra by analyzing the $\mathrm{Ni}$ I 5436 line that is thought to be formed $38 \mathrm{~km}$ above the surface defined by $\tau_{500}=1$. The frequency of the oscillation was found to be $\omega=0.040 \mathrm{~s}^{-1}$. The rms speed of the velocity oscillation at a region of enhanced power was estimated to be $44 \mathrm{~m} \mathrm{~s}^{-1}$, which corresponds to $v_{0}=62 \mathrm{~m}^{-1}$.

Our solution yields the prediction about the wave breaking of the 3 minute waves. The 3 minute waves will develop into shocks at the height of $853 \mathrm{~km}$ above the surface and the velocity amplitude will be $5.4 \mathrm{~km} \mathrm{~s}^{-1}$. We expect that the nonlinear signatures should be observationally detectible from a lower height, say, $600 \mathrm{~km}$ above the surface before the shock is formed.

\subsection{Second-harmonic Signal in the $H \alpha$ Line}

The core of the $\mathrm{H} \alpha$ line is mostly formed in the upper chromosphere. The velocity data used here were obtained by analyzing the cores of the $\mathrm{H} \alpha \mathrm{I}$ line spectra taken from a point inside a pore observed on 2014 June 3 for 68 minutes at the cadence of $20 \mathrm{~s}$. The details of data and analysis were described by Chae et al. (2014).

Three-minute oscillations in the upper chromosphere are well illustrated in the temporal variation of velocity and its wavelet power spectrum presented in Figure 4. It is clear that velocity oscillates with periods of about 3 minutes. The longduration behavior of the observed oscillations, however, differs from the analytical solution given by Equation (63). First, the observed oscillations occur not as an infinite train of waves, but as wave packets of finite duration or wavelets, with the amplitude changing with time. Second, the peak power frequency changes with times.

Nevertheless, the short-duration behavior of the observed oscillations can be fairly well described by Equation (63) because over a short duration, both the amplitude and the peak power frequency can be assumed to be constant. We focus on the behavior during the duration $[49,54]$ minute when the power is the strongest. The fundamental oscillation occurs at periods around 2.8 minute. The most important finding is the existence of the second harmonic with periods around 1.4 minute. This is the first observational finding of the second harmonic theoretically predicted by Litvinenko \& Chae (2017). The second harmonic occurs only when the fundamental oscillation is strong enough, which supports the nonlinear development as its origin.

The right panel of Figure 4 shows the variation of velocity during the short duration. The transition from downward to upward motion is rapid, while that from upward to downward is slow. The figure also shows the result of the model fitting. The fitting is fairly good. The determined parameters are $v_{1}=5.0 \mathrm{~km} \mathrm{~s}^{-1}, \quad P \equiv 2 \pi / \omega=2.8$ minute, and $R_{2}=0.37$. The large values of $v_{1}$ and $R_{2}$ are the characteristics of oscillations in the upper chromosphere.

\subsection{Second-harmonic Signal in the Na I Line}

Figure 5 illustrates 3 minute oscillations of velocity in the low chromosphere seen through the $\mathrm{Na}$ I line. The velocity data points cover 38 minutes at the cadence of $16 \mathrm{~s}$. The $\mathrm{Na} \mathrm{I} \mathrm{D}_{2}$ line spectra were taken from a point inside a sunspot umbra observed on 2015 June 16 . The detailed description of data and analysis can be found in Chae et al. (2017).

From the wavelet power spectrum, we find the existence of the second harmonic in the $\mathrm{Na} \mathrm{I}$ as well. This finding is important because it indicates that the 3 minute oscillations may be significantly nonlinear even in the low chromosphere, not to mention the upper chromosphere.

The model fitting of the short-duration oscillations yielded the values of the parameters: $v_{1}=1.2 \mathrm{~km} \mathrm{~s}^{-1}, P \equiv 2 \pi / \omega=$ 2.74 minute, and $R_{2}=0.19$. Note that the values of $v_{1}$ and $R_{2}$ are smaller than those obtained from the $\mathrm{H} \alpha$ line above, which characterizes the oscillations in the low chromosphere.

\subsection{Comparison of Theory and Observation}

Figure 6 shows the theoretical plots of $R_{2}$ against $\omega / \omega_{0} c_{0}$. It can seen from Equation (62) that $R_{2}$, a measure of nonlinearity, increases with $\omega v_{1}$. We find that in the case of $\omega \simeq \omega_{0}, R_{2}$ 

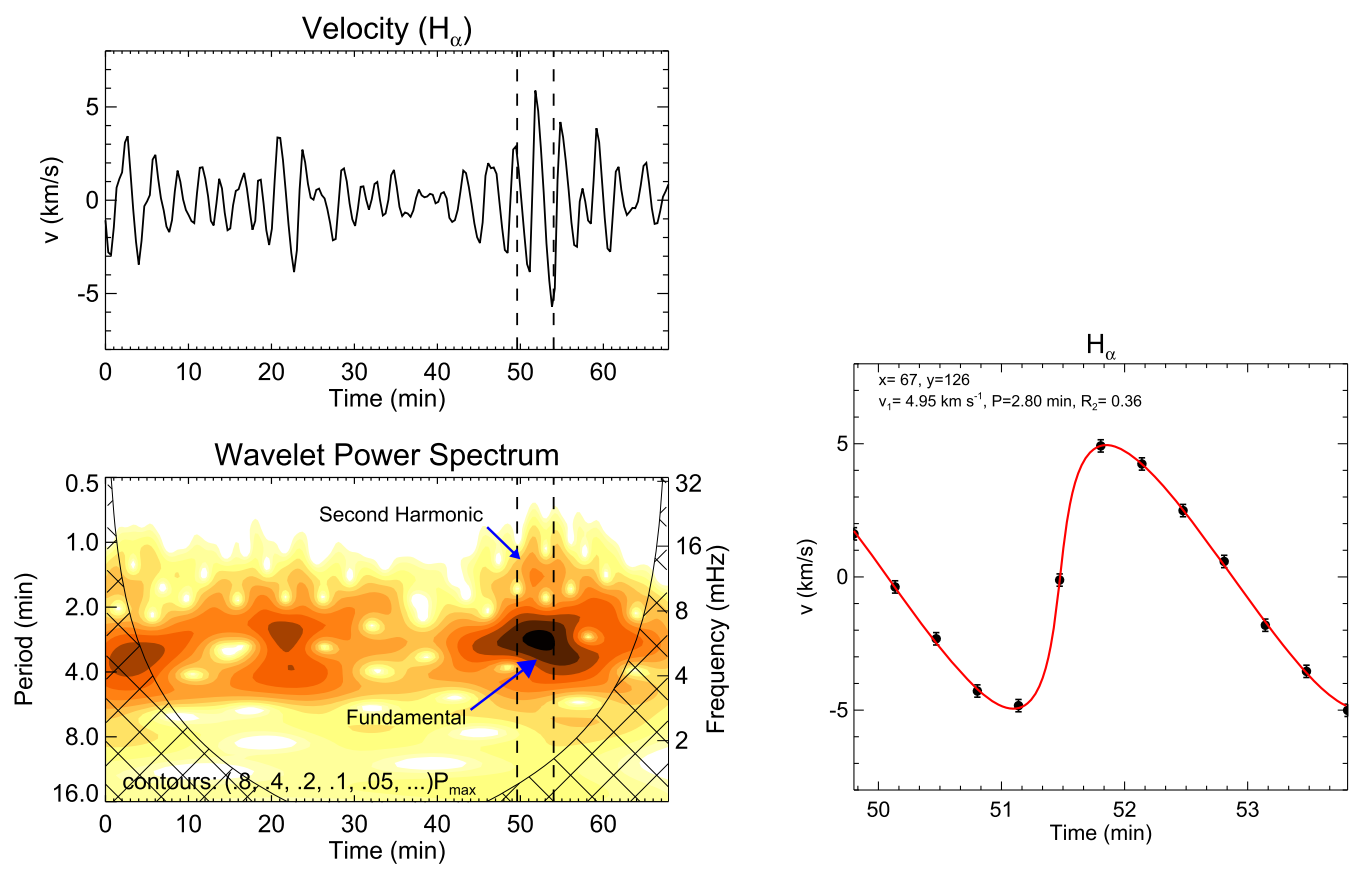

Figure 4. Example of 3 minute oscillations of velocity in the upper chromosphere.

approaches $1 / 2$ as $v_{1}$ approaches $c_{0}$, which of course is consistent with the wave breaking condition, quantified by Equation (54). It is worth stressing that $R_{2}, v_{1}$, and $\omega$ can be determined from observations and $c_{0} \omega_{0}=\gamma g / 2$ can be easily calculated if $\gamma$ is specified. Therefore, the plots in Figure 6 are useful tools for comparing theory and observations.

The two data points obtained from the analysis of observations above were plotted with the plus and diamond symbols for comparison with the theory. We find a qualitatively good agreement between theory and observation. The velocity oscillation measured from the $\mathrm{NaI}$ is moderately nonlinear, with moderate values of $R_{2}$ and $\omega v_{1}$, and the velocity oscillation of the $\mathrm{H} \alpha$ is highly nonlinear with large values of $R_{2}$ and $\omega v_{1}$.

The detailed comparison, however, reveals some discrepancy between theory and observation as well, suggesting the need to elaborate either theory or observation. Supposing the theory is good enough, we can think of reasons why the data points deviate from the theoretical curves. In the $\mathrm{Na}$ I line measurements, there is a possibility that $v_{1}$ may have been underestimated by a factor as large as two since the core of the line is formed not only in the low chromosphere, but also in the photosphere. Meanwhile, the model fitting of the $\mathrm{H} \alpha$ line measurements may have underestimated $R_{2}$ because the temporal sampling is not fine enough to adequately describe the rapid transition from downward to upward motions.

\section{Discussion}

We have derived an analytical solution for the nonlinear propagation of a simple acoustic wave in an isothermal atmosphere that is driven by a sinusoidal motion at the lower boundary. The atmosphere is characterized by sound speed $c_{0}$, gravitational acceleration $g$, and specific ratio $\gamma$. The driving motion is characterized by the angular frequency $\omega$ and amplitude $v_{0}$. In the previous sections, we have presented the solution and analysis using the dimensionless parameters, but here we give a summary of main results by restoring the physical dimensions.

The atmosphere has a pressure scale height $H$ and an acoustic cutoff frequency $\omega_{0}$ that are determined by the values of $c_{0}, g$, and $\gamma$. For the propagation of waves, it is required to have $\omega>\omega_{0}$. We have defined the waves of $\omega>\sqrt{2} \omega_{0}$ as short waves $(\lambda<4 \pi H)$ and the waves of $\sqrt{2} \omega_{0} \geqslant \omega>\omega_{0}$ as long waves $(\lambda>4 \pi H)$. The 3 minute oscillations observed in the solar chromosphere may be identified with the long waves.

Even though we were not able to obtain an exact analytical solution that describes both the short waves and long waves, we have derived an approximate analytical solution applicable to waves of arbitrary wavelength by taking an optimally weighted average of the short-wave solution and the long-wave solution separately determined in two different limits. This combined solution allows us to investigate the continuous transition of the solution from the short waves to the long waves.

The solution we have obtained in the present work is very useful since it is an analytical solution. Even though it has been obtained under the assumption of an isothermal atmosphere and with some approximations, it is good enough not only to describe the nonlinear behavior of the waves (steepening of the wave profile and shock formation), but also to provide reasonable estimates of some physical variables (height of shock formation and velocity amplitude at that height).

We have shown that the steepening of the wave profile is equivalent to the generation of the second harmonic and higher-order harmonics from the fundamental oscillation. The degree of nonlinear development is quantified by $R_{2}$, the amplitude ratio of the second harmonic to the fundamental oscillation. It ranges from zero in the linear stage to $1 / 2$ at the stage of shock formation. The observed temporal variation of velocity at a height can be mathematically modeled by Equation (63) with the four free parameters: amplitude ratio $R_{2}$, amplitude of the fundamental oscillation $v_{1}$, angular frequency $\omega$, and phase $\phi$. The analytical solution we obtained 

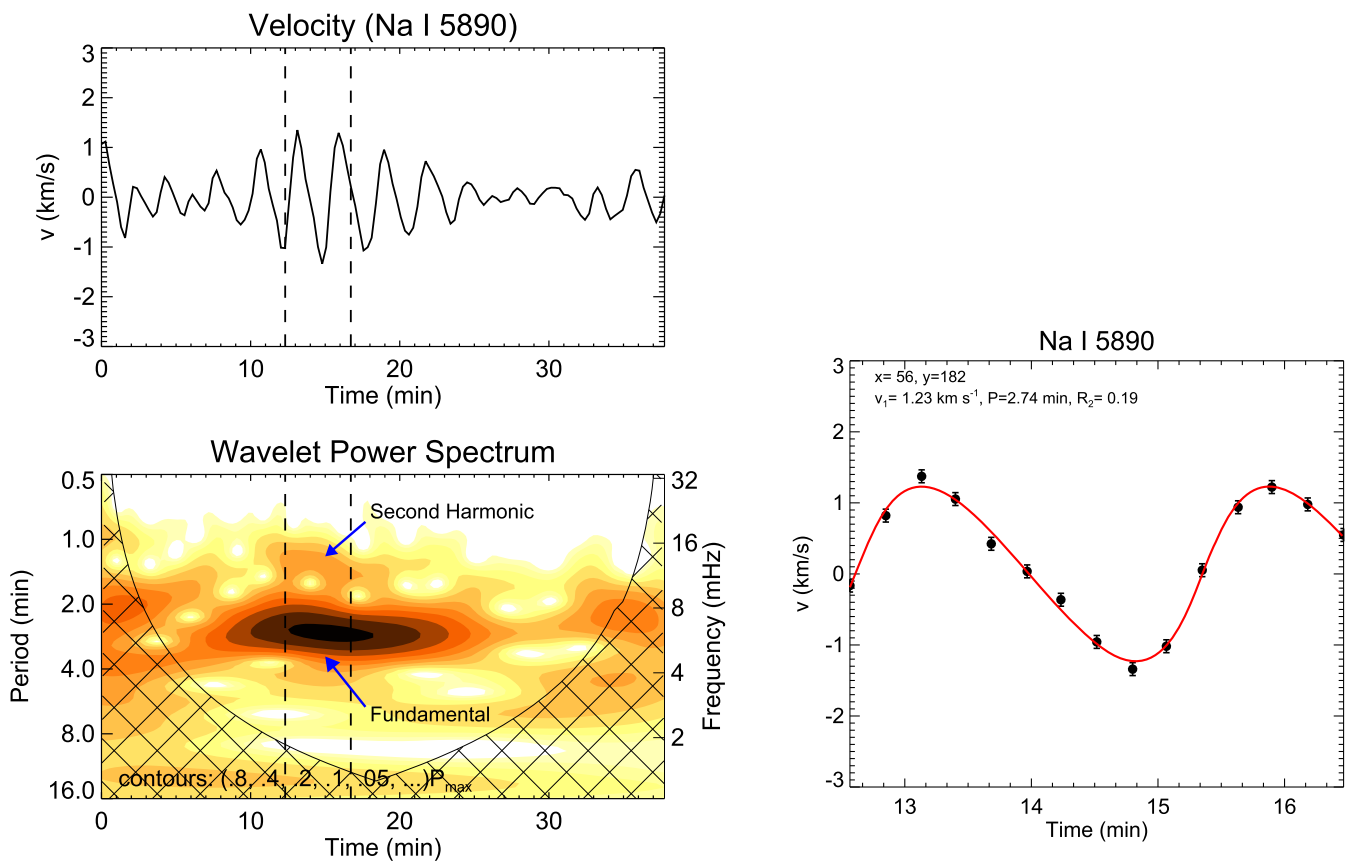

Figure 5. Top: examples of velocity oscillations determined from the $\mathrm{Na}$ I $\mathrm{D}_{2}$ line. Bottom: wavelet power spectra showing both the fundamental oscillations and second-harmonic oscillations.

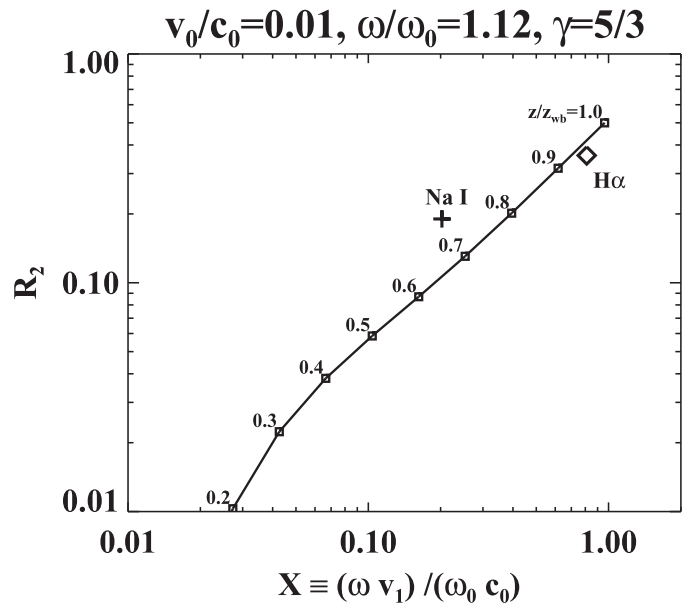

Figure 6. Plots of $R_{2}$ vs. $\omega v_{1} /\left(\omega_{0} c_{0}\right)$ in different cases of $\omega / \omega_{0}$. The two data points were plotted using $\gamma=5 / 3$.

specifies the relationship between $R_{2}$ and $\omega v_{1} / \omega_{0} c_{0}$, which is found to be in rough agreement with the plot of the observationally determined values.

We found that the height of wave breaking or shock formation $z_{\mathrm{wb}}$ is practically determined by $X_{0} \equiv X(0)=$ $\left(\omega / \omega_{0}\right)\left(v_{0} / c_{0}\right)$. Supposing $X_{0} \ll 1$, our solution provides the rule-of-thumb estimates: $z_{\mathrm{wb}} \simeq 2 H \ln \left(\frac{2}{\gamma+2} \frac{1}{X_{0}}\right)$ for the height of shock formation, and $v_{1}\left(z_{\mathrm{wb}}\right) \simeq \frac{2}{\gamma+2} \frac{\omega_{0}}{\omega} c_{0}$ for the velocity amplitude at that height. These are very good estimates for short waves, and, if $\gamma$ is replaced by 1 , for long waves as well.

Finally, we have reported the observational detection of the second harmonic theoretically predicted. We have analyzed the spectral profiles of the $\mathrm{NaI} \mathrm{D}_{2}$ line and the $\mathrm{H} \alpha$ line to determine vertical velocity in the low chromosphere and the upper chromosphere, respectively. The time variation of velocity determined from these lines display the characteristic of the steepening of the wave profile. By applying the mathematical model, we have determined the value of $R_{2}$. This is the first report of the observational determination of $R_{2}$.

Let us think about the validity of the assumption of an initially isothermal atmosphere. We note that even though a real solar atmosphere is not isothermal as a whole, its lower part can be approximated to be isothermal reasonably well. For example, according to the model $\mathrm{M}$ umbral atmosphere of Maltby et al. (1986), the atmosphere is fairly isothermal in the layer from 0 to $800 \mathrm{~km}$ above the photospheric surface. In this layer, temperature ranges between 3500 and $4000 \mathrm{~K}$. Interestingly, the top of this layer coincides with the height of nine pressure scale heights where the 3 minute waves form into shock waves. Since our solution concerns the nonlinear development of the waves from the photosphere up to the height of shock formation, the assumption of isothermal atmosphere is justified in this specific case. For the same reason, we do not have to worry about the effect of shock wave heating. The waves propagate adiabatically below the height of shock formation. Our solution hence should be robust enough and the description of the generation of second harmonic is adequate enough.

In contrast, the behavior of shock waves above the height of shock formation is beyond the scope of our solution. Its physical situation is much more complex than our solution. First, the temperature gradient starts to have a significant effect in the upper atmosphere (Routh \& Musielak 2014; Murawski et al. 2016). Second, the analytical description of shock waves requires a special treatment of discontinuities such as making use of jump conditions in addition to the continuous solution we have obtained. Third, the shock waves heat the atmosphere and can make the temperature structure of the atmosphere deviate from the initial model. This means that in principle the time variation of both the atmospheric structure and the shock wave solution should treated in a consistent way.

We conclude that our work will contribute to the further investigation of nonlinear behaviors of the 3 minute oscillations in the solar chromosphere by linking observation and theory. 
Particularly, the $X-R_{2}$ plot we introduced here may be exploited as a test bed for the comparison between observation and theory. More theoretical works may have to be done to understand the effects of the temperature gradient of the atmosphere, pulse-like driving of finite duration on the nonlinear propagation of the 3 minute waves, and the behavior of shock waves in the upper atmosphere. From the observational side, higher cadence measurements of velocity are required to better determine the amplitude of the second harmonic.

This work was supported by the National Research Foundation of Korea (NRF-2017R1A2B4004466). Y.L.'s contribution was supported by the Ruhr-Universität Bochum Research School PLUS Visiting International Professor Fellowship, funded by Germany's Excellence Initiative (DFG GSC 98/3).

\section{References}

Chae, J., Park, H.-M., Ahn, K., et al. 2013, SoPh, 288, 1 Chae, J., Yang, H., Park, H., et al. 2014, ApJ, 789, 108
Chae, J., \& Goode, P. R. 2015, ApJ, 808, 118

Chae, J., Lee, J., Cho, K., et al. 2017, ApJ, 836, 18

Fawzy, D. E., \& Musielak, Z. E. 2012, MNRAS, 421, 159

Felipe, T., Khomenko, E., Collados, M., \& Beck, C. 2010, ApJ, 722, 131

Fleck, B., \& Schmitz, F. 1991, A\&A, 250, 235

Kalkofen, W., Rossi, P., Bodo, G., \& Massaglia, S. 1994, A\&A, 284, 976

Kalkofen, W., Rossi, P., Bodo, G., \& Massaglia, S. 2010, A\&A, 520, A100

Kwak, H., Chae, J., Song, D., et al. 2016, ApJL, 821, L30

Landau, L. D., \& Lifshitz, E. M. 1969, Mechanics (Oxford: Pergamon)

Lighthill, J. 1978, Waves in Fluids (Cambridge: Cambridge Univ. Press) Litvinenko, Y., \& Chae, J. 2017, A\&A, 599, A15

Maltby, P., Avrett, E. H., Carlsson, M., et al. 1986, ApJ, 306, 284

Murawski, K., Musielak, Z. E., Konkol, P., \& Wiśniewska, A. 2016, ApJ, 827,37

Naugolnykh, K., \& Ostrovsky, L. 1998, Nonlinear Wave Processes in Acoustics (Cambridge: Cambridge Univ. Press)

Nayfeh, A. H. 1975, ASAJ, 57, 1413

Ostrovskii, L. A. 1963, JApMM, 27, 1412

Petukhov, M. Y., \& Petukhov, Y. V. 2001, AstL, 27, 186

Routh, S., \& Musielak, Z. E. 2014, AN, 335, 1043

Sutmann, G., Musielak, Z. E., \& Ulmschneider, P. 1998, A\&A, 340, 556

Tian, H., DeLuca, E., Reeves, K. K., et al. 2014, ApJ, 786, 137

Wiśniewska, A., Musielak, Z. E., Staiger, J., \& Roth, M. 2016, ApJL, 819, L23 